\title{
A!
}

This is an electronic reprint of the original article.

This reprint may differ from the original in pagination and typographic detail.

Zhu, Chao; Mehrabi, Abbas; Xiao, Yu; Wen, Yinghong

\section{CrowdParking: Crowdsourcing based parking navigation in autonomous driving era}

\section{Published in:}

Proceedings of the 2019 21st International Conference on Electromagnetics in Advanced Applications, ICEAA 2019

DOI:

10.1109/ICEAA.2019.8879201

Published: 01/09/2019

Document Version

Peer reviewed version

Please cite the original version:

Zhu, C., Mehrabi, A., Xiao, Y., \& Wen, Y. (2019). CrowdParking: Crowdsourcing based parking navigation in autonomous driving era. In Proceedings of the 2019 21st International Conference on Electromagnetics in Advanced Applications, ICEAA 2019 (pp. 1401-1405). [8879201] IEEE.

https://doi.org/10.1109/ICEAA.2019.8879201

This material is protected by copyright and other intellectual property rights, and duplication or sale of all or part of any of the repository collections is not permitted, except that material may be duplicated by you for your research use or educational purposes in electronic or print form. You must obtain permission for any other use. Electronic or print copies may not be offered, whether for sale or otherwise to anyone who is not an authorised user. 


\title{
CrowdParking: Crowdsourcing Based Parking Navigation in Autonomous Driving Era
}

\author{
Chao Zhu, Abbas Mehrabi, Yu Xiao and Yinghong Wen
}

\begin{abstract}
Finding a free road side parking in urban area is considered as one of the most challenging driving tasks, especially for the autonomous vehicles with limited sight (e.g. short range sensing) and brain (compared with human beings). To assist autonomous vehicle parking in urban area, we propose a novel parking scheme CrowdParking, which applies crowdsourcing and vehicular fog computing to collect parking information from vehicles, locate free parking spaces from crowdsourced data. We also explore the variation of parking availability from a real world data set and find that the availability of specific parking lot has certain relationship with the traffic condition of nearby roads. Based on the observations, we propose the vision of estimating the parking availability with taking into account the traffic condition in neighborhood.
\end{abstract}

\section{INTRODUCTION}

Over the past few years, autonomous vehicles (AVs) capable of identifying relevant signals and obstacles and generating appropriate navigation paths are brought into a reality by the automotive industry (e.g. Audi and BMW) and technology corporations (e.g. Telsa, Uber and Baidu). In autonomous era, parking $\mathrm{AVs}$ at the road side is considered as one of the most challenging driving tasks, where the AVs has to find applicable nearby parking lots by themselves. With the help of advanced sensing (e.g. LIDAR and RIDAR), Automated Valet Parking (AVP) [1] is designed, which allows users to leave their vehicles at the drop-off area, including self-finding vacant parking space, self-driving to the parking lots, and parking themselves at the vacant space. However, due to the limited detection range of sensors (e.g. the range of LIDAR is a few tens of meters [2]), the vacant parking lots can be detected only if the AVs are traveling approach to them, whereas that located outside the detection range of sensors cannot be spotted. Therefore, the extra traffic may be caused while AVs are aimlessly cruising for vacant parking spots in urban area.

With the growing adoption of dash-camera, few works saw the potential for innovations by collecting the video from vehicles and detecting vacant parking lots by video processing [3], [4], [5]. Compared to burying sensors under the pavement beneath the slotted parking spaces, recognizing free

C. Zhu, A. Mehrabi, Y. Xiao are with Aalto University, Espoo 02150 Finland. E-mail: \{chao.1.zhu, abbas.mehrabidavoodabadi, yu.xiao\} @aalto.fi

Y. Wen is with Beijing Engineering Center of EMC and GNSS Technology for Rail Transportation and School of Electronic and Information Engineering, Beijing Jiaotong University, Beijing, 100044, China.

E-mail: yhwen@bjtu.edu.cn

This research work was partially funded by the Beijing Science Program of Beijing Municipal Science and Technology (No.Z181100001018032) and Academic of Finland(317432). parking spaces from crowdsourced video by object recognition technology is a more economic solution. However, heavyweight video processing algorithms can not be implemented on individual vehicle due to its limited computing capacity, while the remarkable transmission delay and huge volume of data traffic will be generated when uploading the crowdsourced video stream to the servers located at cloud.

To conform the vehicular environment where operations are latency-critical, a novel computing paradigm called vehicular fog computing (VFC) [6], [7] has been proposed. The terms of VFC refers to push large amount of intelligence (i.e., computing and communicating resources) to the fog nodes (e.g. roadside small cells) near where the vehicular application tasks are being generated. With shorter physical distance and less communication hops, the data generated by vehicular applications can be transmitted with low latency. Furthermore, by caching the content of local common interest (e.g. the processed results of tasks) in fog nodes, the processing latency of tasks demanding for local-aware service can be reduced. In this paper, we design a novel parking scheme CrowdParking, applying video crowdsourcing to collect video stream from vehicles and VFC to process parking analytic data, to get the real-time parking information in urban area and assist AVs to find available parking spaces quickly. In CrowdParking, we turn small cells and commercial fleets into fog nodes. With large amount of computing resources equipped, complex clustering based video processing algorithms can be implemented on fog nodes for locating the vacant parking lots and an optimal parking navigation can be provided for AVs. Beside visual-based parking analytic data, such information of magnetized parking lots also can be crowdsourced from vehicles with magnetic sensors.

In reality, the parking lots assumed to be free may be fully occupied when the AV arrives. To avoid the AVs being "blocked", we split roads into small segments and analyze the parking availability (e.g. the probability that the AVs are blocked) of each segment, taking into account the number of vacant parking lots and traffic condition of the neighborhood. Compared with disseminating the location of individual vacant parking lot, informing the AVs about such information of the road segments enhances chance of a successful parking. After known the location of the optimal parking road segment, the AVs will be navigated to the destination and self-find a free parking lot while cruising the road segment.

\section{SySTEM OVERVIEW}

In this section, we present related terms and introduce the procedure of task offloading. 


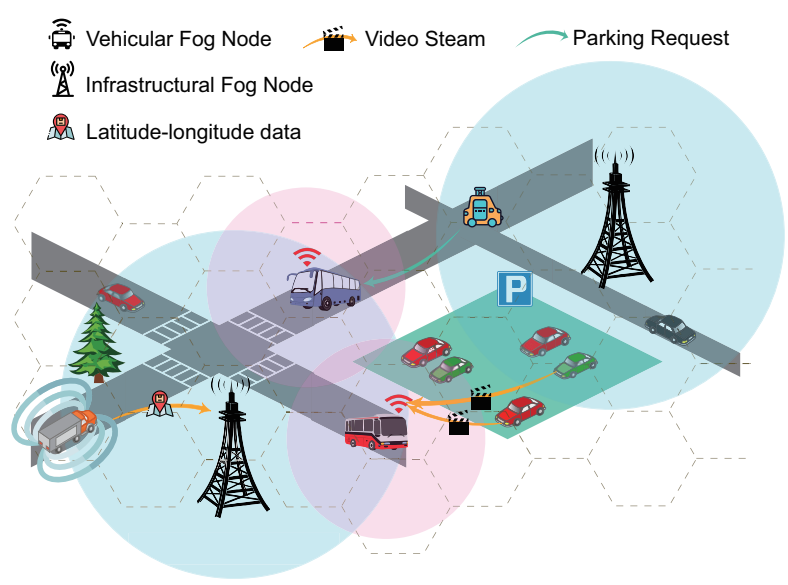

Fig. 1: Architecture of CrowdParking

\section{A. Related Terms}

1) Participant Vehicles: In CrowdParking, any vehicle with visual-based (e.g. dash-camera) or magnetic sensors on board, and certain amount of computing resources can be acted as a participant vehicle. For participant vehicles equipped with dash-camera, they capture and process video stream, and detect vacant road-side parking lots. Furthermore, the magnetized parking lots can be detected by participant vehicles with magnetic sensors, utilizing the Earths magnetic field. Once any vacant parking lot is detected, the client vehicle will report to fog nodes and upload the parking analytic data.

2) Client Vehicles: The AVs that request the information about available surrounding parking lots are defined as client vehicles. The client vehicles are enabled with the ability of self-finding and self-parking at a vacant parking lot which is located within limited distance.

3) Fog Nodes: The fog nodes are responsible for collecting requests from client vehicles, aggregating the parking analytic data that the participant vehicles upload and estimating the availability of parking lots. We consider two types of fog nodes.

- Infrastructure Fog nodes: The computing nodes colocated with network infrastructures (i.e., small cells).

- Vehicular Fog nodes: The computing nodes carried by moving public transportations (buses) with on-board opportunistic (e.g. WiFi, bluetooth and DSRC [8]) and cellular communication modules.

\section{B. Process of CrowdParking}

In CrowdParking, the participant vehicles detect vacant road-side parking lots while traveling in urban area, and upload the video stream to surrounding fog nodes once any available parking lot is detected. With aggregating and caching the parking analytic data, fog nodes estimate the availability of parking lots and inform the client vehicle about the optimal parking location, taking into account the traffic situation and travel cost.

1) Segmenting Roads: As illustrated in Fig.1, we split the roads in urban area into small segments with spatial matrix (represented by a grid map consisting of divided grid cells on the area of interest) and each road segment may contain certain number of parking lots. In CrowdParking, a client vehicle will be informed of the location of the road segment which is most applicable for parking, rather than the precise location of available parking lots. Once the location of the road segment is acquired, the client vehicle will travel to that area and find an available parking lot by itself.

2) Detecting Parking Lots: In CrowdParking, participant vehicles with dash-camera captures video and locally processing frames in real-time, looks at the road-side parking spaces. The participant vehicles apply light-weight machine learning techniques, such as the Viola-Jones feature-based cascade classifier [9], to detect parking lots in the dashcameras video stream. Furthermore, the real-time location of participant vehicles, e.g. GPS, will be embedded in the video stream.

For the participant vehicles with magnetic sensors, they detect vacant parking lots by the perceiving the variation of surrounding Earths magnetic field, and then report their current location and number of vacant to fog nodes in real time.

3) Locating and Counting Parking Lots: Due to the short coverage of magnetic sensors, only vacant parking lots which are close to participant vehicles can be detected. Thus, the location of participant vehicles can be equivalent to the location of detected parking lots. However, this method does not work for the participant vehicles with dash-camera, because vacant parking lots located far away from participant vehicles also can be detected from video stream. Due to the limitation of computing capacity, the detection algorithm applied in the participant vehicles only provides the position of the parking lots in the coordinates of the cameras frame. To estimate parking lots latitude-longitude positions in the real world, fog nodes apply complex clustering algorithm, e.g. DBSCAN [10], to map from camera coordinates to a three-dimensional road coordinate system and count the number of vacant parking lots on the road segment.

4) Parking Availability Estimation: The availability of parking lots changes with time and the parking lots may be fully occupied when the client vehicle arrives at the destination road segment. We use a blocking probability to denote the probability that a vehicle could be blocked, i.e., the parking lots are full when the vehicle arrives. To provide a friendly parking information dissemination, fog nodes will estimate the blocking probability of road segments, taking into account the number of vacant parking lots and the traffic condition of surrounding area, which has a great impact on the travel time of client vehicles and the birth-death of parking lots (the death of a parking lot means that a vehicle enters and occupies the parking lot, while the birth of a parking lot means that the vehicle leaves the parking lot and the parking lot becomes vacant).

\section{VISION OF PARKING AVAILABILITY Estimation}

In this section, we introduce the primary method for parking availability estimation, which predict the number of vacant roadside parking lots in the near future, according to the traffic condition of surrounding area. More specifically, we try to 


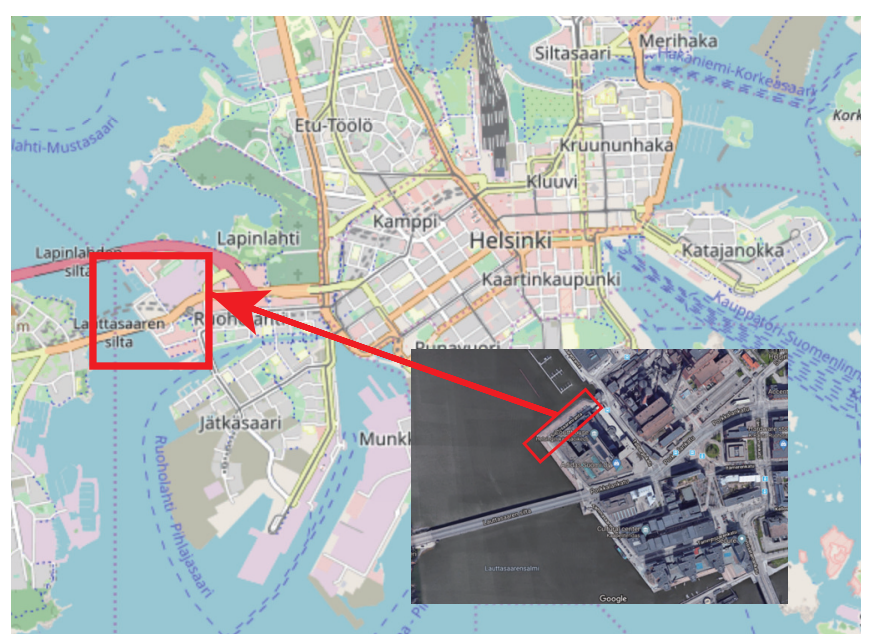

Fig. 2: Location of The Parking Lot

explore the impact of the traffic condition (average speed, incidents and traffic jam) on the availability of nearby parking lots.

\section{A. Datasets}

In this work, we collected the traffic dataset and the parking dataset. The traffic dataset consists of traffic road network and, traffic jam and traffic speed sub-dataset, collected from HERE API [11]. The parking data set accumulates information about parking ticket machines and all private mobile payment operators in Helsinki. From the parking data set, we choose a specific parking lot which located at $(60.16415,24.90026)$ for the case study. The parking lot contains 20 parking spots and its location on the Open Street Map is shown in Fig.2.

In the traffic dataset, HERE divides every road into several segments. We collected the traffic information of the road segments which are located nearby (within $300 \mathrm{~m}$ and $500 \mathrm{~m}$ ) the specific parking lot for about 4 weeks $(19$ th $\sim 22$ th Week, 2019). Furthermore, we recorded the variation of the vacant parking spots in the specific parking lot during the same period.

\section{B. Vacant Parking Spots Variation}

Fig.3 shows the variation of the number of parked vehicles in the specific parking lot during four weeks (19th $\sim 22$ th Week, 2019). On each Monday morning, we can see that the number of parked vehicles keeps increasing from 7:00 to

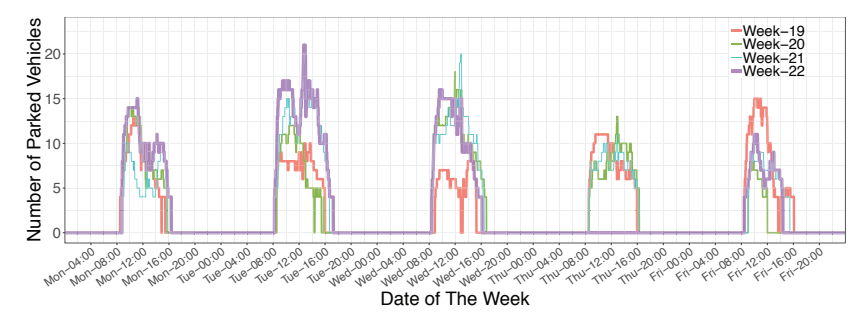

Fig. 3: Variation of The Number of Parked Vehicles

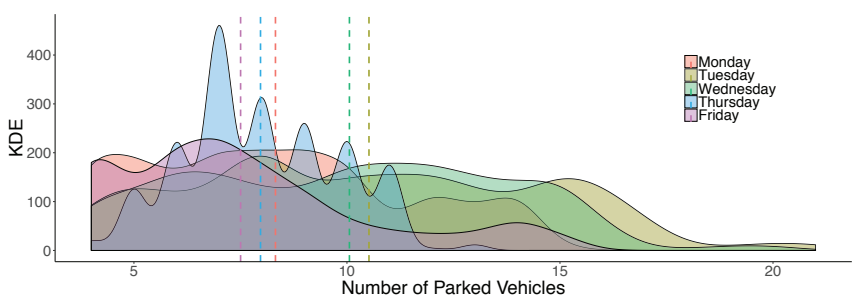

Fig. 4: Distribution of The Number of Parked Vehicles

10:00. From 10:00 to 11:00, the number of parked vehicles stays above 12 . This may be people working in this area start driving to work in the morning. After 11:00, the number of parked vehicles starts decreasing and drops to $8 \sim 10$ around 12:00.

On the Tuesday and Wednesday of each week, we can see the number of parked vehicles stays at a higher level compared with other dates of the week. This may be because these two days are in the middle of working days and people have entered the working state. On the Thursday and Friday of each week, we can see the number of parked vehicles stays at a lower level. This may be because the passion of peoples work retreats as the weekend approaches. Notably, the Thursday of the 22th Week is the Ascension Day in Finland and there is no parking data recorded in that date.

We analyze the kernel density estimation (KDE) of the number of parked vehicles in each working day in Fig.4. We can see the average number of parked vehicles on Tuesday and Wednesday is above 10. Among all the working days, the number of parked vehicles on Friday reaches the minimum number, which is around 8. From the Fig.4, we can see that the availability of parking spots is higher on the days near the weekends, and vice versa.

\section{Nearby Traffic Condition Variation}

To identify the temporal variation of vehicular traffic condition, we collected the traffic message channel in a region of 67 square kilometers in Helsinki using Here Traffic API [11] for about one month from 6rd May to 2th June, 2019. The TMC data contains the traffic reports received from vehicles, including the location, direction, average speed of the traffic flow. From the TMC data, we can get the jam factor, which represents the expected quality of travel. The jam factor is a number between 0 and 10 . The larger the jam factor is, the higher the vehicular traffic density is. From the data set, we extract the temporal variation of the jam factor of four road segments which are located within 300 meters of the specific parking lot and illustrate it in Fig.5. We try to explore whether there exist certain relationship between and the variation of the jam factor and that of the availability of the parking lot.

In the Fig.5, we can see there exists certain pattern in variation of jam factor of each road in the temporal aspect. For example, compared with road III and road IV, the jam factor of the road I and road II does not have a strong variation. Furthermore, the jam factor of each road stays at a lower level on the Thursday and Friday compared with that on the Tuesday and Wednesday. This share the same trend with the temporal 
-Week-19-Week-20-Week-21-Week-22

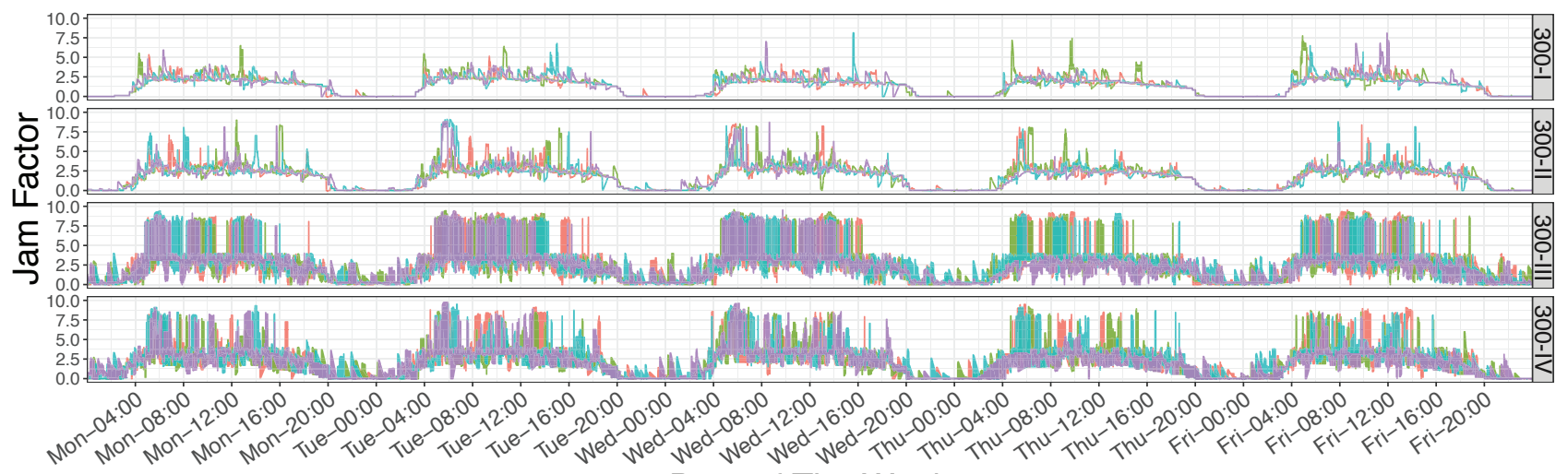

Date of The Week

Fig. 5: Variation of The Jam Factor of Roads within 300 m

variation of the selected parking lot. Beside that, the jam factor of each road start growing at around 07:00 in the morning on each working day. After about one hour later, the number of the parked vehicles also start growing. We can see that the availability of parking lot is related to the traffic condition of the nearby roads.

\section{Vision of Traffic Condition and Parking Availability Cor- related Time Series Forecasting}

From the Fig.3 and Fig.5, we can see there exists certain relationship between the parking availability and the traffic condition of nearby road. Therefore, accurate forecasting of correlated time series of the traffic condition of the nearby roads have the potential to reveal the underlying holistic dynamics of the availability of specific parking lot, including identifying trends, predicting future parking availability, and detecting anomalies, which are important to enable effective operations of the traffic condition of nearby roads.

To enable accurate and robust correlated time series forecasting, two non-linear forecasting algorithms based on deep neural networks, a Convolutional Recurrent Neural Network (CRNN) and an Auto-Encoder Convolutional Recurrent Neural Network (AECRNN), can be used [12]. In CRNNs, we can first consider the variation of parking availability and that of nearby roads' traffic condition independently and feed each time series into a 1-dimensional convolutional neural network $(\mathrm{CNN})$. In AECRNNs, we can add additional auto-encoders into CRNN. The output of convoluted time series are not only merged together to be fed into the RNN. In addition, each convoluted time series is also reconstructed back to the original time series. Then, the objective function combines the error of the prediction by the RNN and the reconstruction discrepancy by the auto-encoders, making AECRNN a multi-task learning model.

\section{RELATED WORK}

In this section, we first describe the work related to crowdsourcing based parking navigation, and then introduce the appliance of fog computing in the vehicular networking.

\section{A. Crowdsouring Based Parking Navigation}

In the past few years, plenty of works [13], [14], [15], [16] have beendevoted to the system design and implementation of crowdsourcing based parking navigation. Cben et al. [13] took smart parking as a case study for mobile crowdsourcing and simulated this specific application scenario. Hara et al. [14] investigated the feasibility of using untrained crowd workers from Amazon Mechanical Turk (turkers) to find, label, and assess sidewalk parking spots in Google Street View imagery. Shi et al. collected and disseminated parking space information based on crowd knowledge, and made estimation of future availability of the parking spaces by information aggregating. Rajasegarar et al. [16] analysed the parking occupancy data collected from real-world data set and employed various prediction mechanisms, such as machine learning, for the parking occupancy rate.

\section{B. Vehicular Fog Computing}

Due to the space, weight, and cost constraints, computing capacity of most vehicles may not be high enough to handle image/video processing tasks, such as vacant parking spot detection. On the other hand, offloading these tasks to the cloud is not applicable, due to the remarkable transmission delay. Thus, the concept of vehicular fog computing has been proposed and widely explored by plenty of works [18], [19], [20], [21], [22]. Xiao et al. [19] proposed to turn commercial fleets into fog nodes to serve neighboring vehicles and passengers, while Hou et al. [18] suggested utilizing the extra computing power on slow moving or parked vehicles. Zhu et al. [20] proposed to utilize fog computing to collect and process video for certain vehicular applications, such as parking detection. Furthermore, Zhu et at. [21], [22] designed a system for latency and performance balanced task offloading for video transmission and processing in vehicular networking.

\section{CONCLUSION}

In this paper, we propose CrowdParking, a novel parking scheme designed to assist AVs to find an applicable parking 
space in urban area. We split road into segments, collect parking information with crowdsourcing and locate parking lots with implementing complex video processing technologies in fog nodes. Furthermore, we also explore the variation of parking availability from a real world data set and find that the availability of specific parking lot has certain relationship with the traffic condition of nearby roads. Based on the observations, we propose the vision of estimating the parking availability with taking into account the traffic condition in neighborhood to avoid AV's to be blocked.

\section{REFERENCES}

[1] H. Banzhaf, D. Nienhser, S. Knoop, and J. M. Zllner, "The future of parking: A survey on automated valet parking with an outlook on high density parking," in 2017 IEEE Intelligent Vehicles Symposium (IV), June 2017, pp. 1827-1834.

[2] J. Hecht, "Lidar for self-driving cars," Optics and Photonics News, vol. 29, no. 1, pp. 26-33, 2018

[3] C. Zhu, G. Pastor, Y. Xiao, and A. Ylajaaski, "Vehicular fog computing for video crowdsourcing: Applications, feasibility, and challenges," IEEE Communications Magazine, vol. 56, no. 10, pp. 58-63, 2018.

[4] G. Grassi, K. Jamieson, P. Bahl, and G. Pau, "Parkmaster: An invehicle, edge-based video analytics service for detecting open parking spaces in urban environments," in Proceedings of the Second ACM/IEEE Symposium on Edge Computing. ACM, 2017, p. 16.

[5] J. Ni, A. Zhang, X. Lin, and X. S. Shen, "Security, privacy, and fairness in fog-based vehicular crowdsensing," IEEE Communications Magazine, vol. 55, no. 6, pp. 146-152, 2017.

[6] Y. Xiao and C. Zhu, "Vehicular fog computing: Vision and challenges," in 2017 IEEE International Conference on Pervasive Computing and Communications Workshops (PerCom Workshops), March 2017, pp. 69.

[7] C. Zhu, G. Pastor, Y. Xiao, Y. Li, and A. Ylä-Jääski, "Fog following me: Latency and quality balanced task allocation in vehicular fog computing," in 2018 15th Annual IEEE International Conference on Sensing, Communication, and Networking (SECON). IEEE, 2018, pp. $1-9$.

[8] J. B. Kenney, "Dedicated short-range communications (dsrc) standards in the united states," Proceedings of the IEEE, vol. 99, no. 7, pp. 1162 $1182,2011$.

[9] R. Lienhart and J. Maydt, "An extended set of haar-like features for rapid object detection," in Proceedings. International Conference on Image Processing, vol. 1. IEEE, 2002, pp. I-I.

[10] M. Ester, H.-P. Kriegel, J. Sander, X. Xu et al., "A density-based algorithm for discovering clusters in large spatial databases with noise." in $K d d$, vol. 96, no. 34, 1996, pp. 226-231.

[11] "Here API." [Online]. Available: https://developer.here.com/documentation/traffic/ [Accessed Sep. 01, 2018]

[12] R.-G. Cirstea, D.-V. Micu, G.-M. Muresan, C. Guo, and B. Yang, "Correlated time series forecasting using deep neural networks: A summary of results." [Online]. Available: http://arxiv.org/abs/1808.09794

[13] X. Chen, E. Santos-Neto, and M. Ripeanu, "Crowdsourcing for on-street smart parking," in Proceedings of the Second ACM International Symposium on Design and Analysis of Intelligent Vehicular Networks and Applications, ser. DIVANet '12. ACM, pp. 1-8, event-place: Paphos, Cyprus. [Online]. Available: http://doi.acm.org/10.1145/2386958.2386960

[14] K. Hara, V. Le, and J. Froehlich, "Combining crowdsourcing and google street view to identify street-level accessibility problems,' in Proceedings of the SIGCHI Conference on Human Factors in Computing Systems - CHI '13. ACM Press, p. 631. [Online] Available: http://dl.acm.org/citation.cfm?doid=2470654.2470744

[15] F. Shi, D. Wu, D. I. Arkhipov, Q. Liu, A. C. Regan, and J. A. McCann, "ParkCrowd: Reliable crowdsensing for aggregation and dissemination of parking space information," pp. 1-13.

[16] a. S. Rajasegarar and C. Leckie, "Parking availability prediction for sensor-enabled car parks in smart cities," in 2015 IEEE Tenth International Conference on Intelligent Sensors, Sensor Networks and Information Processing (ISSNIP), pp. 1-6.

[17] C. Huang, R. Lu, and K. R. Choo, "Vehicular fog computing: Architecture, use case, and security and forensic challenges," vol. 55, no. 11, pp. 105-111.
[18] X. Hou, Y. Li, M. Chen, D. Wu, D. Jin, and S. Chen, "Vehicular fog computing: A viewpoint of vehicles as the infrastructures," vol. 65 , no. 6 , pp. 3860-3873.

[19] Y. Xiao and C. Zhu, "Vehicular fog computing: Vision and challenges," in 2017 IEEE International Conference on Pervasive Computing and Communications Workshops (PerCom Workshops), pp. 6-9.

[20] C. Zhu, G. Pastor, Y. Xiao, and A. Ylajaaski, "Vehicular fog computing for video crowdsourcing: Applications, feasibility, and challenges," vol. 56, no. 10 , pp. 58-63.

[21] C. Zhu, J. Tao, G. Pastor, Y. Xiao, Y. Ji, Q. Zhou, Y. Li, and A. Yl-Jski, "Folo: Latency and quality optimized task allocation in vehicular fog computing," pp. 1-1.

[22] C. Zhu, G. Pastor, Y. Xiao, Y. Li, and A. Ylae-Jaeaeski, "Fog following me: Latency and quality balanced task allocation in vehicular fog computing," in 2018 15th Annual IEEE International Conference on Sensing, Communication, and Networking (SECON), pp. 1-9. 